\title{
Late Instability Following Total Hip Arthroplasty
}

\author{
Luis Pulido, MD; Camilo Restrepo, MD; and Javad Parvizi MD, FRCS
}

\begin{abstract}
Instability is one of the most common complications after total hip arthroplasty and can present early or late after hip replacement. Late instability is considered if the event occurs five or more years after the primary arthroplasty, and in contrast to early dislocation, it appears to require operative intervention. The incidence of late instability may be greater than initially appreciated, and the cumulative rate rises with longer follow-up. The etiology of hip instability is often multifactorial with the presumed risk factors for late instability including long standing malposition of the components, trauma, deterioration in muscle mass, neurological status impairment and polyethylene wear. This article presents a synopsis of published studies on late instability and outlines our institutional experience with treatment of late dislocation following total hip arthroplasty occurring due to polyethylene wear.
\end{abstract}

Keywords: Dislocation; Hip; Arthroplasty; Joint instability; Postoperative complications; Prosthesis failure

$\mathrm{H}$ reason for individuals to seek medical attention. The symptoms of arthritis may be addressed by non-operative management delivered by primary care physicians; ${ }^{1}$ however, for patients failing non-operative treatment, total hip arthroplasty (THA) is considered. THA is one of the most successful procedures in orthopaedic surgery that has effectively improved the quality of life of patients affected by osteoarthritis of the hip. ${ }^{2}$ Unfortunately the outcome of THA is compromised by complications such as instability. ${ }^{3}$ Instability comprises two inter-related yet distinct conditions, dislocation and subluxation. Dislocation is a term reserved for situations in which the femoral head is completely out of the acetabulum, whereas subluxation is partial dislocation in which the femoral head is partially out of the acetabulum.

Instability can present early or late after hip replacement. ${ }^{4,5}$ Late dislocation is considered if the event occurs five or more years after the primary arthroplasty, and in contrast to early dislocation, it appears to require operative intervention. Recent studies suggest that the incidence of late dislocation may be greater than initially appreciated ${ }^{4,6-8}$ and that the cumulative rate rises with longer follow up. ${ }^{9}$
In this article, we review the etiological factors for late instability. We also present our institutional experience on late instability due to polyethylene wear as the primary cause when no discrete reason for dislocation is identified.

\section{Etiology}

The etiology of hip instability is often multifactorial. Patient demographics, operative technique and implant design variables have been demonstrated to affect the risk of dislocation. There are multiple surgical and implant-related factors that predispose to dislocation, such as malorientation of the components, decreased head-to-neck ratio, proximal placement of the acetabular component, decreased femoral offset, posterior surgical approach, abductor insufficiency secondary to trochanteric migration, or abductor avulsion. 3,5,10-12 Patient risk factors for early dislocation after primary THA are female gender, older patients, neurological dysfunction or cognitive impairment, and a preoperative diagnosis of osteonecrosis of the femoral head, femoral neck fracture and/or inflammatory arthritis. $3,9,10,12$ The presumed etiological factors for late instability include long standing malpositioning of the components, trauma, deterioration in muscle mass, neurological status impairment and polyethylene wear $4,5,13$ (table 1).
Reprint Requests: Javad Parvizi MD, Rothman Institute, 925 Chestnut Street, Philadelphia, PA 19107, Tel: 267339 3617, Fax: 215- 503 0580, Email: parvj@aol.com
Received:August 16, 2006

Revised: December 28, 2006

Accepted: January 18, 2007 
Table 1. Risk factors for late instability following total hip arthroplasty.

Female gender

Younger age

Pseudocapsule laxity

Abductors mechanism insufficiency

Previous subluxations

Hip trauma

Cognitive impairment

Neuromuscular dysfunction

Initial malposition of the acetabular component

Loosening with migration of the components

Polyethylene wear

\section{Presentation}

Instability following THA is a frequent and very distressful complication. It may occur early or late, and can present as a single or recurrent event. If the prosthesis dislocates early after surgery, there is a higher rate of success for closed reduction, as opposed to late dislocation in which revision arthroplasty surgery is most likely required. ${ }^{4}$ Late instability can be a challenging problem as it is associated with a higher risk of recurrent dislocation and rate of reoperation. ${ }^{4,12,14}$

\section{Prevalence}

The prevalence of dislocation following THA is likely underestimated. A close surveillance and a more rigorous follow-up for a longer period is likely to identify more late dislocations. ${ }^{4,9,11}$ The cumulative risk of a first-time dislocation progressively increases with time: $1 \%$ at 1 month, $1.9 \%$ at 1 year, a constant rate of $1 \%$ every 5 years, and a $7 \%$ dislocation rate at 25 years. ${ }^{9}$ Late dislocation is more common than previously thought, as one third of all the dislocated hips occur five or more years after the primary replacement. ${ }^{4}$ Berry et $\mathrm{al}^{9}$ reported a $4.8 \%$ prevalence rate of dislocation after 6,623 consecutive primary Charnley THAs over 15 years.

\section{Institutional Experience}

We have previously reported on the association between polyethylene wear and late dislocation of the hip. ${ }^{13,15}$ Twenty-two patients with late dislocation and polyethylene wear (measuring $>2 \mathrm{~mm}$ ) were retrospectively evaluated (figure 1). Patients with onset of instability at less than 5 years postoperatively, those with radiographic and/or intraoperative evidence of component malpositioning, and patients with any previous history of prosthetic hip instability were excluded. Analysis of the medical records and radiographs were performed.

The cohort consisted of 7 women and 15 men with a mean age of 57.8 years (range 35-85) at initial primary arthroplasty. The revision surgery was performed between the years 1982 and 2000. The average time from initial arthroplasty to dislocation was 9.0 years. Revision surgery to address polyethylene wear and instability was performed at a mean of 11.1 years (range 5.8 to 23 years). Direct lateral approach was used in all cases.

Acetabular liner was exchanged in 11 of the 22 patients. The liner was cemented into the well-fixed and well-positioned cup in two hips, and inserted into a functioning locking mechanism in nine hips. The remaining 11 patients with all polyethylene, cemented acetabular component required revision of the acetabular component. The initial acetabular component was confirmed to be well-positioned intraoperatively. An uncemented acetabular component was inserted press-fit for all these patients. The femoral head was changed in all cases. Five of the 22 patients, who were noticed to have osteolysis around their cemented prosthesis, underwent revision of their femoral component using an extensively coated component. Revision surgery restored stability to 18 patients $(82 \%)$, with a redislocation rate of $18 \%$ $(4 / 22)$. Although the patients who underwent acetabular liner exchange did not present any episodes of instability during follow-up, it is important to highlight that all the cases had also received femoral head exchange. Furthermore, the femoral head was upsized from 22 to $28 \mathrm{~mm}$ in three patients and the neck length increased in an additional three patients.

\section{Discussion}

The success of THA in restoring function and alleviating pain cannot be disputed. The number of primary hip replacements per year in the United States is rising dramatically, and this trend is expected to continue at an exponential rate. ${ }^{16}$ Therefore, primary care physicians, as well as orthopedic surgeons, are likely to encounter more patients in need of THA.

Although very successful, THA may fall victim to complications such as instability. Instability after THA can occur after $2 \%$ to $5 \%$ of primary hip surgeries. ${ }^{3}$ Late instability represents one-third of all dislocations. ${ }^{4}$ Coventry ${ }^{7}$ described that patients with late dislocation had a larger range of motion and attributed pseudocapsule laxity as a possible etiological factor for late dislocation.

A recent study from the same institution delineated the causes of late dislocation after 19,680 primary THAs over a period of 25 years. They identified a total of 165 hips which had the first dislocation event five or more years after the primary arthroplasty. ${ }^{4}$ Factors associated with a higher risk of late dislocation included female gender and a younger age at the time of operation. One-fourth of their cohort presented with previous subluxations, substantial trauma or marked neurological impairment. ${ }^{4}$ Surgical or implant-related factors such as initial malposition of the acetabular component, loosening with migration or change in position of the components, or a femoral head penetration of more than 2 $\mathrm{mm}$ into the acetabular polyethylene were radiographically identified in $34 \%$ of these patients. ${ }^{4}$ Late dislocation recurred 

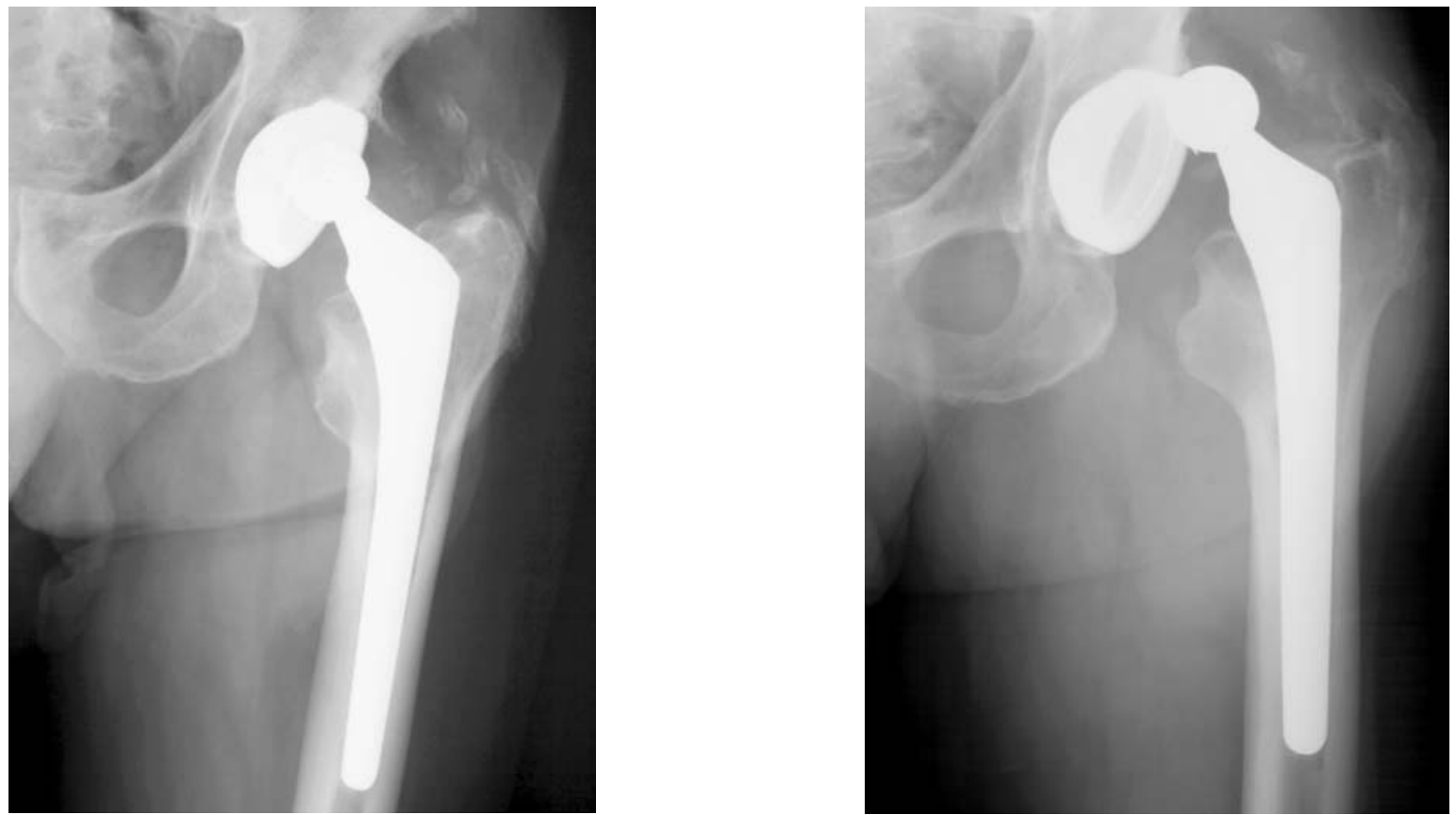

Figure 1. Anterior-posterior radiograph of the left hip in a 68-year-old patient. (A) Polyethylene wear is present and can be identified by eccentric seating of the femoral head in the acetabulum. (B) Polyethylene wear was implicated as the main cause of late instability in this patient.

in $55 \%$ of the patients, and $61 \%$ of these recurrent dislocations required surgical intervention for treatment.

Polyethylene wear is an important cause of late instability (figure 1). Primary care physicians need to be familiar with radiographic evaluation and detection of liner wear in order to seek an early intervention. This condition can be identified by eccentric seating of the femoral head in the acetabular component (figure 1). Once identified, these patients can receive surgical intervention that may prevent complications such as late instability and osteolysis.

The exact mechanism by which polyethylene wear results in late instability remains unknown. It has been suggested that polyethylene wear debris eliciting an inflammatory response may result in capsular distension and subsequent instability. ${ }^{7}$ On the other hand, eccentric seating of the femoral head with asymmetric excursion arc of motion may impart a mechanical disadvantage predisposing the hip to impingement, levering out and subsequent dislocation. Another factor is that polyethylene-wear presenting many years after the initial surgery occurs in the context of an aging patient with gradual deterioration in the status of the periarticular muscle mass and the dynamic stabilizers. The critical importance of soft tissues, particularly the abductors, in stabilizing the hip cannot be overemphasized.

The treatment of late dislocation as a result of polyethylene wear by liner exchange or revision of the all-polyethylene acetabular component is a viable option that can be expected to provide significant improvement in function and stabilize the hip in most patients.

\section{References}

1. Karpman RR. Musculoskeletal disease in the United States: who provides the care? Clin Orthop Relat Res 2001;385:52-56.

2. Felson DT, Lawrence RC, Hochberg MC, McAlindon T, Dieppe PA, Minor MA, Blair SN, Berman BM, Fries JF, Weinberger M, Lorig KR, Jacobs JJ, Goldberg V. Osteoarthritis: new insights. Part 2: treatment approaches. Ann Intern Med 2000;133:726-737.

3. Morrey BF. Instability after total hip arthroplasty. Orthop Clin North Am 1992;23:237-248.

4. von Knoch M, Berry DJ, Harmsen WS, Morrey BF. Late dislocation after total hip arthroplasty. J Bone Joint Surg Am 2002;84-A:1949-1953.

5. Paterno SA, Lachiewicz PF, Kelley SS. The influence of patient-related factors and the position of the acetabular component on the rate of dislocation after total hip replacement. J Bone Joint Surg Am 1997;79:1202-1210.

6. Callaghan JJ, Heithoff BE, Goetz DD, Sullivan PM, Pedersen DR, Johnston RC. Prevention of dislocation after hip arthroplasty: lessons from long-term followup. Clin Orthop Relat Res 2001;393:157-162.

7. Coventry MB. Late dislocations in patients with Charnley total hip arthroplasty. J Bone Joint Surg Am 1985;67:832-841.

8. Dorr LD, Wan Z. Causes of and treatment protocol for instability of total hip replacement. Clin Orthop Relat Res 1998;355:144-151.

9. Berry DJ, von Knoch M, Schleck CD, Harmsen WS. The cumulative long-term risk of dislocation after primary Charnley total hip arthroplasty. J Bone Joint Surg Am 2004;86-A:9-14.

10. Ali Khan MA, Brakenbury PH, Reynolds IS. Dislocation following total hip replacement. J Bone Joint Surg Br 1981;63-B:214-218.

11. Sanchez-Sotelo J, Berry DJ. Epidemiology of instability after total hip replacement. Orthop Clin North Am 2001;32:543-552, vii.

12. Woolson ST, Rahimtoola ZO. Risk factors for dislocation during the first 3 months after primary total hip replacement. J Arthroplasty 1999;14:662-668. 
13. Parvizi J, Wade FA, Rapuri V, Springer BD, Berry DJ, Hozack WJ. Revision hip arthroplasty for late instability secondary to polyethylene wear. Clin Orthop Relat Res 2006;447:66-69.

14. Sanchez-Sotelo J, Haidukewych GJ, Boberg CJ. Hospital cost of dislocation after primary total hip arthroplasty. J Bone Joint Surg Am 2006;88:290-294.

15. Orozco F, Hozack WJ. Late dislocations after cementless total hip arthroplasty resulting from polyethylene wear. J Arthroplasty 2000;15:1059-1063.

16. Kurtz S, Mowat F, Ong K, Chan N, Lau E, Halpern M. Prevalence of primary and revision total hip and knee arthroplasty in the United States from 1990 through 2002. J Bone Joint Surg Am 2005;87:1487-1497.

\section{Author Affiliations}

Luis Pulido, MD

Rothman Institute of Orthopedics

Philadelphia, Pennsylvania, USA

Camilo Restrepo, $M D$

Rothman Institute of Orthopedics

Philadelphia, Pennsylvania, USA

Javad Parvizi MD, FRCS

Rothman Institute of Orthopedics

Philadelphia, Pennsylvania, USA

Correction

\section{Evaluation of Genetic Factors for Warfarin Dose Prediction (Clin Med Res 2007;5:8- I6. doi: I 0.3 I 2 I/cmr.2007.724)}

Michael D. Caldwell, MD, PhD; Richard L. Berg, MS; Kai Qi Zhang, BS; Ingrid Glurich, PhD; John R. Schmelzer, PhD; Steven H. Yale, MD; Humberto J. Vidaillet, MD; and James K. Burmester, PhD†

The original article to which this correction refers was published in the March 2007 issue of Clinical Medicine \& Research (Clin Med Res 2007;5:8-16. doi:10.3121/cmr.2007.724).

The authors reported the groups were incorrectly labeled in the originally published version of Figure $1 \mathrm{~A}$. The correct group labels are (left to right) $* 1 / * 1, * 1 / * 2, * 1 / * 3, * 2 / * 2, * 2 / * 3, * 3 / * 3$. These are the same groups that are further partitioned in Figure 1B. A corrected figure is provided here (see corrected Figure 1A).

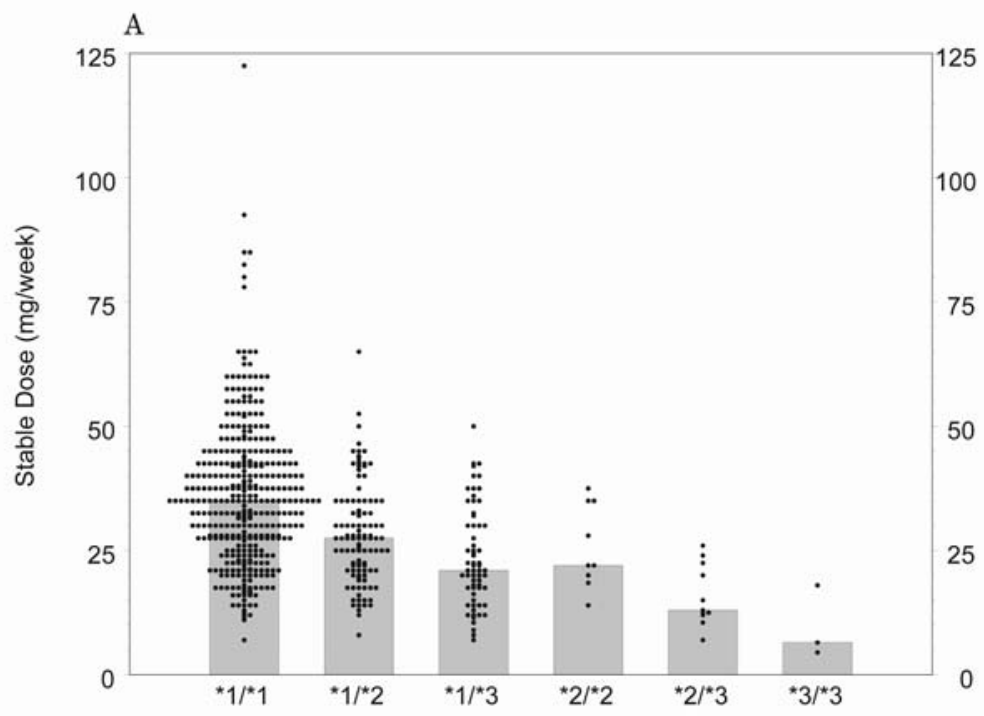

Correction Figure 1A. Stable coumadin dose by genotype. Distribution of weekly stabilizing warfarin dose in relation to (A) CYP2C9 and (B) CYP2C9 and VKORC1 genotypes. The CYP2C9 genotype is labeled as ${ }^{*} 1 /{ }^{*} 1,{ }^{*} 1 /{ }^{*} 2,{ }^{*} 1 /{ }^{*} 3,{ }^{*} 2 /{ }^{*} 2,{ }^{*} 2 /{ }^{*} 3$ and ${ }^{*} 3 /{ }^{*} 3$. For $(B)$, within each CYP2C9 genotype, the VKORC1 genotype is shown as GG, GC or CC. [see original Figure 1 to view panel B].

† Address correspondence to: James K. Burmester, PhD; Center for Human Genetics, Marshfield Clinic Research Foundation, I000

North Oak Avenue, Marshfield,WI 54449.Telephone: 7I5-389-4368; Fax: 7I5-389-3808; Email:burmester.jim@mcrf.mfldclin.edu.

doi: $10.3121 / \mathrm{cmr}$.2007.724.corr 